\title{
L'arbitrage entreprise / marché : le rôle du contrôle interne, outil de réduction des coûts de transaction
}

\author{
Eustache Ebondo (*) \& Benoît PigÉ (**)
}

(*) Professeur au Groupe ESC Marseille-Provence.

(**) Professeur agrégé des Universités en Sciences de Gestion, membre du L.I.B.R.E (Université de Franche-Comté).

Les auteurs remercient les deux rapporteurs de la revue ainsi que les participants au congrès de l'AFC à Metz en 2001 pour leurs observations et remarques.

Avril 2002 


\title{
L'arbitrage entreprise / marché : le rôle du contrôle interne, outil de réduction des coûts de transaction
}

\author{
The choice firm / market: the importance of internal control as a mean \\ to reduce the transaction costs
}

\section{Résumé}

Dans l'étude des organisations, la distinction entre le marché et l'entreprise apparaît fondée, depuis 1937 et l'article de Coase, sur l'économie des coûts de transactions. L'avantage de l'entreprise est d'être capable de gérer certaines transactions à un coût moindre que le marché, en raison notamment de l'existence de systèmes de contrôle interne qui permettent une meilleure prise en compte des spécificités des transactions et assurent une limitation de la latitude discrétionnaire de chacun en présence d'une asymétrie d'information. Le développement des nouvelles technologies de l'information et de la communication, cumulé avec un accroissement des connaissances et une spécialisation des savoirs, entraîne la nécessité de repenser la notion d'organisation en fonction des avantages comparatifs de celle-ci, avantages fondés sur les mécanismes de contrôle interne existants ou à mettre en œuvre.

Mots clés : coûts de transaction, contrôle interne, asymétrie d'information

\section{Summary}

Since 1937 and the Coase article, the distinction between the enterprise and the market seems to repose on the costs transaction economy. The enterprise advantage is of being able to manage some transactions at a lesser cost than the market, due to the existence of internal control mechanisms which authorize a better appreciation of transactions specificity and allow a limitation of opportunism when there is information asymmetry. The new information and communication technologies' development, cumulated with the augmentation and the specialization of the knowledge, induces the need to think again the distinction between the firm and the market and specifically the internal control mechanisms which are to be implemented.

Keywords : transaction costs, internal control, information asymmetry 
Depuis Coase (1937), la distinction entre les organisations et le marché apparait fondée sur la notion de coûts de transaction tels qu'ils ont été définis par Arrow (1969) puis par Williamson (1985). L'avènement de ce qui est qualifié de " nouvelle économie " tend parfois à affecter cette distinction traditionnelle en modifiant, sinon la nature, du moins le montant des coûts de transaction concernés. Les entreprises, mais de manière générale toutes les organisations, sont amenées à repenser les limites de leur structure organisationnelle: quelles sont les activités qui sont constitutives de leur existence et quelles sont les activités qui peuvent être externalisées et sous-traitées au marché?

Dans cet article, nous nous proposons de remettre en évidence le rôle des mécanismes de contrôle interne dans la performance relative des organisations face au marché. En effet, ce n'est pas l'un des moindre paradoxe de l'essor de l'économie Internet que de soulever les nombreux problèmes liés aux risques de perte de contrôle de l'information et de la communication (le développement des courtiers en ligne repose ainsi sur la confiance dans la sécurité et la confidentialité des transactions réalisées en direct depuis son ordinateur). Si la structure organisationnelle évolue, il devient indispensable de faire évoluer en parallèle le système de contrôle interne, ou de contrôle organisationnel, qui en assure la cohérence.

Dans les sections ci-après, nous replaçons ces principaux enjeux par rapport à la distinction entre le marché et l'entreprise. Nous estimons que les procédures de contrôle interne constituent un élément clé pour déterminer le niveau des coûts en vigueur au sein de l'entreprise, par rapport aux coûts encourus pour la réalisation de ces mêmes transactions sur le marché. Il apparaît alors nécessaire de s'interroger sur les différents mécanismes de contrôle interne qui permettent de minimiser les coûts de transaction internes à l'organisation.

La prise en compte de l'impact organisationnel des nouvelles technologies de l'information et de la communication, mais aussi et surtout des nouvelles approches en matière de gestion des compétences, conduit à repenser certaines procédures de contrôle interne. Chaque entreprise est ainsi amenée à identifier ses activités créatrices de valeur. Parmi elles, l'activité de contrôle interne apparaît comme constitutive de l'organisation dans sa différence par rapport au marché.

\section{La distinction marché et entreprise : le rôle central du contrôle interne}

Si la théorie des coûts de transaction permet d'expliquer la distinction entre le marché et l'entreprise, les facteurs sur lesquels reposent ces coûts de transaction sont néanmoins sujets à 
variation. Il convient donc de déterminer les coûts associés aux différentes composantes des coûts de transaction.

\subsection{L'économie des coûts de transaction}

A la suite de la démarche de Coase (1937), on peut estimer que, d'un point de vue économique, les entreprises se distinguent des marchés par leur capacité à internaliser certaines transactions et à les réaliser à un coût moindre que si elles avaient dû se dérouler sur les marchés.

Williamson (1985) a formalisé le coût de ces transactions en distinguant selon qu'ils sont préalables ou postérieurs à la conclusion de la transaction. Ainsi, il définit les coûts de transaction ex ante comme étant les coûts associés à la rédaction, à la négociation et à la garantie de l'accord obtenu. Les coûts de transaction ex post sont définis comme: les coûts de mauvaise adaptation, dus au fait que les transactions se désajustent; les coûts de marchandage, occasionnés si des efforts bilatéraux sont faits pour corriger des divergences ex post; les coûts d'organisation et de fonctionnement, associés aux structures de gouvernance dans lesquelles les conflits sont traités ; les coûts d'établissement d'engagements certains.

\subsection{Les trois dimensions des transactions}

Selon Williamson, certains types de transaction se prêtent davantage que d'autres à leur réalisation au sein d'une entreprise par rapport à leur réalisation sur le marché. Si l'entreprise peut parfois être plus efficiente que les marchés, c'est qu'elle dispose de procédures de contrôle interne lui permettant de faire face au moindre coût aux trois dimensions des transactions que sont :

- La spécificité des actifs : un actif est dit spécifique lorsque sa valeur dans des utilisations alternatives est plus faible que dans son utilisation présente. La spécificité des actifs, attribut essentiel de la transaction (Alchian, 1984), est génératrice de forts coûts de transaction. En effet, plus la spécificité des actifs croît, plus l'investisseur est vulnérable ex post au comportement de son partenaire, plus, en conséquence, il a besoin de garanties ex ante pour consentir à investir dans de tels actifs.

- L'incertitude: elle recouvre plusieurs réalités (Gabrié et Jacquier 1994). La conception qui nous intéresse est l'incertitude comportementale, c'est-à-dire l'incertitude qui porte, non pas sur des événements extérieurs, mais sur le 
comportement futur des agents impliqués dans la transaction, face à des événements qui n'auront pu être intégralement envisagés lors de l'initialisation de la transaction.

- La fréquence: dès lors qu'une transaction est appelée à se répéter, les parties concernées auront intérêt à s'engager dans un contrat à long terme remplaçant une série de contrats ponctuels, afin d'éviter les coûts de renégociation et de réécriture des contrats à chaque occurrence.

\subsection{L'arbitrage entre l'entreprise et le marché}

La spécificité des actifs, l'incertitude sur le déroulement des contrats et la fréquence des transactions conduisent à internaliser les transactions (schéma 1). En effet, en raison du risque de comportement opportuniste des différents partenaires, il est nécessaire, soit de recourir à des clauses contractuelles spécifiques pour modéliser le comportement de chacun ou prévoir des modes de résolution des conflits, soit d'internaliser les transactions en laissant aux procédures de contrôle interne le soin de limiter ces mêmes risques. 
Schéma 1 : Coûts de transaction et contrôle interne

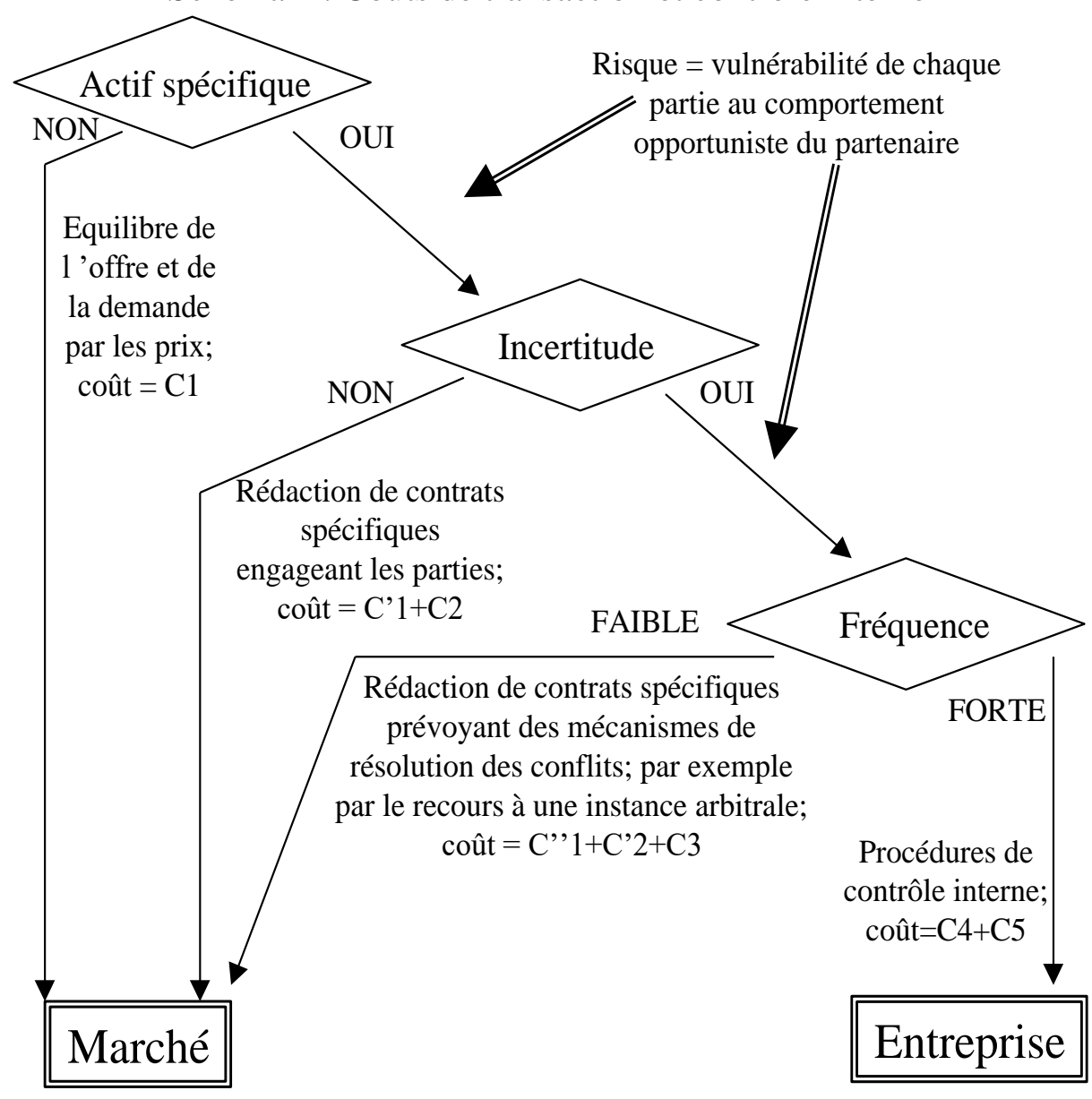

Légende:

$\mathrm{C} 1=$ Coût d'information sur le marché

$\mathrm{C} 2=$ Coût de rédaction, négociation et garantie

$\mathrm{C} 3=$ Coût de résolution

C4=Coût de décision

C5= Coût d'exécution

Source: Pigé (2001), p.27.

Selon la théorie des coûts de transaction, l'entreprise sera préférée au marché quand elle permettra de réaliser certaines transactions à un coût moindre, ce qui dans notre schéma 1 s'écrit : C4+C5 < C', $1+\mathrm{C}$ '2+C3, avec :

$\mathrm{C} 1 \leq \mathrm{C}$ ' $1 \leq \mathrm{C}$ ''1 : le coût d'information sur le marché est d'autant plus faible qu'il n'y a pas d'incertitude et que les actifs sont communs (non spécifiques);

et $\mathrm{C} 2 \leq \mathrm{C}^{\prime} 2$ : le coût de rédaction, de négociation et de garantie des contrats est d'autant plus faible que l'incertitude est elle-même faible. 


\section{Le rôle du contrôle interne dans la maîtrise des coûts de transaction}

La maîtrise des coûts C4 et C5 (coûts de décision et d'exécution internes à l'entreprise) peut donc permettre d'expliquer, en partie, le recours à l'entreprise comme lieu de réalisation de certaines transactions. Or, cette maîtrise des coûts va dépendre en premier lieu de l'existence du contrôle interne et des procédures qui vont être mises en place.

\subsection{Les objectifs du contrôle interne}

Les objectifs du contrôle interne sont au nombre de trois :

- S'assurer que les décisions prises sont correctement appliquées: en effet, l'internalisation des transactions suppose qu'il existe un mécanisme interne permettant de faire ajuster l'offre et la demande internes autrement que par le seul mécanisme des prix ;

- Garantir un niveau minimum de qualité à la prestation effectuée ou au produit fabriqué : le marché permet normalement un ajustement de l'offre et de la demande par les prix en fonction de la qualité du service ou du produit offert. Ainsi, sur le marché pétrolier, le cours du baril coté correspond à une qualité bien spécifique de pétrole. Selon la nature du pétrole échangé, le prix spot fixé sur le marché sera ajusté pour tenir compte des caractéristiques techniques différentes. En interne, il est nécessaire de disposer de procédures de contrôle permettant de s'assurer que les spécificités du produit ou du service correspondent à ce qui était demandé ;

- Déceler les anomalies de fonctionnement : sur le marché, un fournisseur de biens ou services qui connaît des dysfonctionnements internes est automatiquement éliminé car il n'est plus capable de répondre à la demande aux conditions définies par la concurrence. Au sein d'une entreprise, des procédures doivent permettre d'identifier et de corriger les dysfonctionnements de l'organisation pour permettre à cette dernière de remplir ses fonctions de manière efficiente. Ces procédures de contrôle interne doivent aussi, et surtout, éviter que les dysfonctionnements localement observés sur la réalisation d'une transaction donnée ne se traduisent par la mise en faillite de l'entreprise dans sa globalité.

Chacun de ces objectifs est lié à un coût de transaction et entraîne la mise en place et l'application d'un certain nombre de procédures de contrôle interne (schéma 2). 
Schéma 2 : Objectifs du contrôle interne et procédures à mettre en place

Objectif

\begin{tabular}{|c|}
\hline S'assurer que les \\
décisions prises \\
sont correctement \\
appliquées \\
\hline
\end{tabular}

Coût

correspondant
Procédures de

contrôle interne
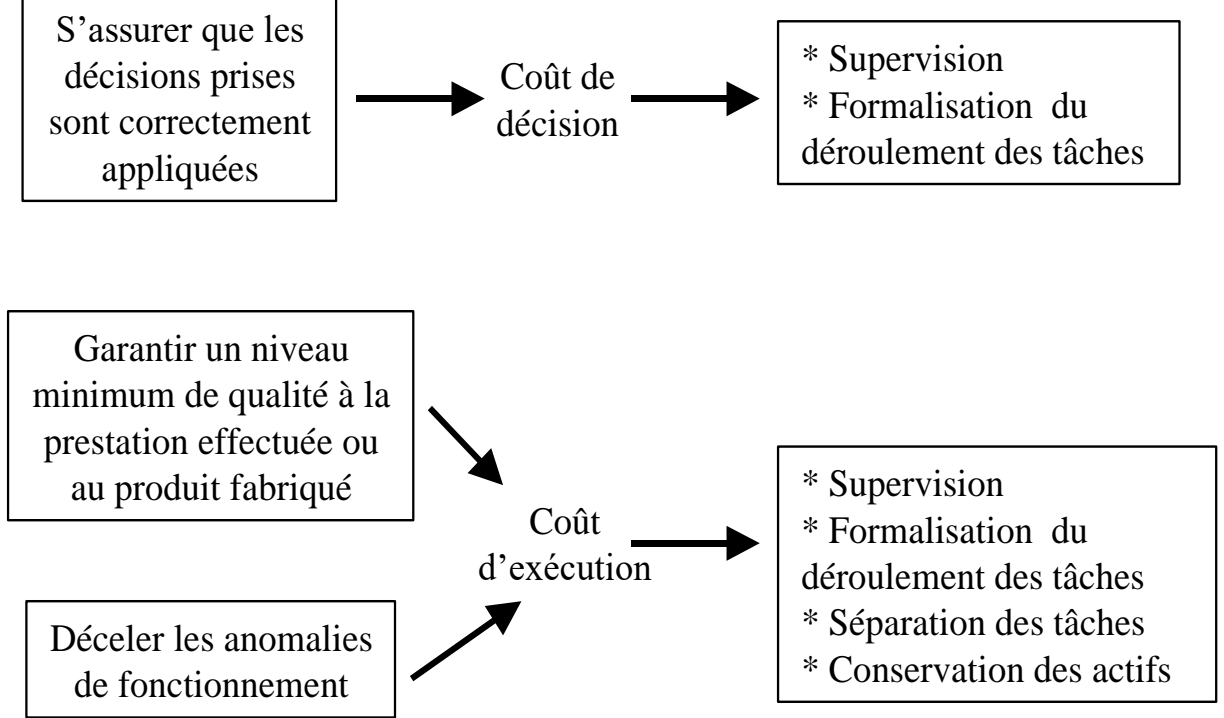

Source: Pigé (2001), p.38.

\subsection{Les procédures de contrôle interne visant à minimiser les coûts de transaction}

Quatre grands types de procédures de contrôle interne (Mikol 1991, Collins et Valin 1992, Becour et Bouquin 1996, Renard 1997) vont contribuer à réduire les coûts de transaction (schéma 2).

\subsubsection{La supervision}

$\mathrm{Si}$, dans les petites entreprises, le dirigeant peut assurer l'essentiel des tâches de décision, le problème de la délégation de pouvoirs et de l'existence de mécanismes de contrôle des décisions décentralisées se pose avec la croissance de la taille organisationnelle de l'entreprise. En effet, "la délégation de pouvoirs signifie que la direction centrale abandonne le contrôle de certaines décisions à des niveaux hiérarchiques inférieurs, c'est-àdire aux personnes plus directement impliquées dans les transactions quotidiennes" (Coopers \& Lybrand 1994, p.42). Les procédures de supervision doivent permettre de s'assurer que les personnes directement impliquées dans les transactions quotidiennes ont pris des décisions conformes à l'intérêt global de l'entreprise.

\subsubsection{La formalisation du déroulement des tâches}

Alors que, sur le marché, toute transaction passe par un échange, dans l'entreprise, les transactions sont intégrées les unes aux autres et leur bon déroulement nécessite que chaque 
acteur de l'entreprise sache exactement ce qu'il doit faire. La vision de Fritz Lang dans son film Metropolis (années 1920) explicite très clairement cette notion où l'on voit un travailleur dont la seule tâche est d'actionner une manette en fonction de l'affichage d'un cadran de contrôle. Simplement, cette manette, qui pour lui n'a aucune signification pratique, conditionne le fonctionnement de l'ensemble du système de production. La formalisation du déroulement des tâches se donne ainsi pour objet de coordonner des actions, a priori disparates, pour en faire un tout cohérent et coordonné (Desreumaux 1997).

\subsubsection{La séparation des tâches.}

Du point de vue du contrôle interne, le principe de séparation des tâches consiste à attribuer, à des personnes ou à des services distincts, des fonctions qui, si elles étaient accomplies par la même personne ou le même service, augmenteraient les risques d'erreurs ou de fraude. On distingue ainsi trois phases critiques dans le déroulement d'une transaction :

- l'autorisation ou l'initialisation de la transaction ;

- la manipulation et le contrôle des actifs liés à la transaction ;

- l'enregistrement de la transaction.

Un bon contrôle interne suppose que chacune de ces phases de la transaction soit confiée à une personne distincte.

\subsubsection{La conservation des actifs}

La réalisation des transactions sur le marché suppose que chaque acteur perçoit directement le fruit de son travail. De même, les actifs qu'il possède constituent son patrimoine professionnel et, à ce titre, il en prend soin. Quand les transactions sont internalisées, le patrimoine productif perd son affectation personnelle. Ce patrimoine est alors susceptible d'être moins bien entretenu et il est nécessaire d'établir des procédures visant à le conserver en bon état de fonctionnement pour l'entreprise. Il en est de même des actifs financiers ou incorporels.

\subsection{L'alternative acquisition ou location : internalisation ou externalisation des transactions, le coût du contrôle interne}

Le succès d'une entreprise de location de matériel comme Kiloutou est intéressant à examiner car il pose bien le problème des coûts associés à toute transaction. Kiloutou offre à tout entrepreneur, ou à tout particulier, la possibilité de louer pour un temps déterminé un 
matériel que ce même client pourrait autrement acquérir. Le prix de facturation marginal est dégressif en fonction du temps.

Tableau 1: Exemple de tarif en fonction des durées de location ${ }^{\mathrm{i}}$

\begin{tabular}{|l|r|r|r|r|r|}
\hline & $\begin{array}{c}\text { 4h ou } \\
\text { forfait nuit }\end{array}$ & $\begin{array}{c}\text { Les 1 ères } \\
24 \mathrm{~h} \\
(1)\end{array}$ & $\begin{array}{c}\text { 24h } \\
\text { suivantes } \\
(2)\end{array}$ & $\begin{array}{c}\text { Forfait 1 } \\
\text { semaine }\end{array}$ & $\begin{array}{c}\text { Durée supérieure à 7 } \\
\text { jours (par jour ouvrable): } \\
(3)\end{array}$ \\
\hline Matériel 1 & 255 & 314 & 281 & 932 & 140 \\
\hline Matériel 2 & 1501 & 2002 & 1892 & 8103 & 1502 \\
\hline
\end{tabular}

Tableau 2: Estimation des coûts liés à la transaction

\begin{tabular}{|l|r|r|r|r|}
\hline & $\begin{array}{c}\text { (1)-(2) } \\
\text { en valeur }\end{array}$ & $\begin{array}{c}\text { (1)-(2) } \\
\text { en \% de (2) }\end{array}$ & $\begin{array}{c}\text { (2)-(3) } \\
\text { en valeur }\end{array}$ & $\begin{array}{r}\text { (2)-(3) } \\
\text { en \% de (3) }\end{array}$ \\
\hline Matériel 1: M1 & 33 & $12 \%$ & 141 & $101 \%$ \\
\hline Matériel 2: M2 & 120 & $6 \%$ & 390 & $26 \%$ \\
\hline
\end{tabular}

La fonction économique de facturation en fonction du temps, $\mathrm{P}_{\mathrm{Mi}}(\mathrm{t})$, est donc de la forme :

$P_{M i}(t)=F_{0}(M i)+F_{1}(M i ; t)+F_{2}(M i) \times t$

avec $\forall(i, t), F_{0}(M i)>0 ; F_{1}(M i ; t)>0 ; F_{2}(M i)>0$

$\frac{\delta P_{M i}(t)}{\delta t}>0$ (la facturation globale est croissante en fonction de la durée de location)

et $\frac{\delta P_{M i}(t)}{\delta \delta t} \leq 0$ (la dérivée seconde est négative ou nulle : la facturation marginale est décroissante en fonction de la durée de location).

$F_{0}(M i)$ couvre les coûts d'engagement liés à la transaction. Notre évaluation de ces coûts correspond à la colonne [(1)-(2)] du tableau 2.

$F_{1}(M i ; t)+F_{2}(M i) \times t$ correspond, pour l'entreprise de location, au prix à percevoir (en fonction de la durée de location) pour couvrir l'usure du matériel (son remplacement à prévoir), sa maintenance (les équipements à remplacer, les consommables éventuels à rajouter, ...), la marge à dégager et les coûts d'exécution de la transaction. Tous ces éléments sont évalués en tenant compte de la durée estimée de vie du matériel et de son temps d'utilisation (le matériel n'est bien évidemment pas loué en permanence).

Quels sont les coûts liés à l'exécution de la transaction ? Il s'agit en fait des coûts liés à l'absence de contrôle interne, ou plutôt à l'absence de garanties sur l'existence, chez l'utilisateur, de procédures de contrôle interne portant sur la conservation des actifs ${ }^{\mathrm{ii}}$. L'utilisateur d'un actif loué n'aura pas les mêmes soins pour le matériel que s'il en était propriétaire. L'entreprise propriétaire du matériel en est consciente et facture donc un surcoût 
pour sa location. Notre évaluation de ce surcoût correspond à la colonne [(2)-(3)] du tableau 2 , c'est à dire à: $F_{1}(M i ; t)$.

Comment cette transaction peut-elle évoluer? Si l'utilisateur a un besoin fréquent du matériel, la solution la plus simple est alors l'acquisition du matériel, puisque l'utilisateur mettra en œuvre des procédures de contrôle interne qui auront un coût inférieur au prix facturé par l'entreprise de location. Dans notre exemple, si l'utilisateur utilise à 10 reprises, au cours de la période étudiée, le même matériel pour une durée récurrente de 2 jours, l'acquisition pourra être pertinente si les coûts de contrôle spécifiquement supportés sont inférieurs àiii: $F_{0}(M i)+F_{1}(M i ; t)$, soit

- $10 \times 33+10 \times(2 \times 141)=3.150$ pour le premier matériel ;

- $10 \times 120+10 \times(2 \times 390)=9.000$ pour le second matériel .

L'autre solution est le développement d'une relation marchande où l'utilisateur accepte de mettre en place certains coûts de contrôle en échange d'une réduction du prix de facturation. Cette solution est particulièrement pertinente quand les transactions sont fréquentes mais que le matériel considéré change.

Ce renouvellement des transactions explique l'existence d'un double tarif selon la qualité du client. Le tarif pour les particuliers intègre la facturation totale des coûts de contrôle (et est donc plus élevé), alors que le tarif pour les artisans intègre une facturation seulement partielle des coûts de contrôle (l'artisan est supposé contrôler l'utilisation du matériel loué au même titre que l'utilisation de son propre matériel). L'artisan étant amené à effectuer de nombreuses transactions avec l'entreprise de location, il a intérêt à supporter certains coûts de contrôle pour obtenir globalement une réduction de son prix de facturation. La fonction de coût de l'utilisateur est de la forme:

$\mathrm{C}_{\text {utilisation }}=\mathrm{P}_{\mathrm{Mi}}(\mathrm{t})+\mathrm{b} \times(\mathrm{C} 4+\mathrm{C} 5)$

avec $(\mathrm{C} 4+\mathrm{C} 5)$ les coûts de contrôle interne chez l'utilisateur

et $\mathrm{b}[0 \leq b \leq 1]$ la proportion de ces coûts de contrôle que l'utilisateur est prêt à supporter en échange d'une réduction du prix PV(t) facturé par l'entreprise de location.

L'arbitrage entre l'internalisation des transactions et le recours au marché dépendra donc de nombreux facteurs parmi lesquels:

- le montant des ressources spécifiques à consacrer au contrôle interne chez l'utilisateur;

- le risque de détérioration (ou d'usure accélérée) du matériel lié à une absence de contrôle qui déterminera le prix de facturation par l'entreprise locatrice. 


\section{La nouvelle économie et son incidence sur la distinction entreprise et marché}

La nouvelle révolution économique, à laquelle nous assistons à ce changement de siècle, a de nombreuses conséquences, notamment en matière de remise en cause de procédures de contrôle interne auparavant considérées comme acquises (schéma 3). En contrepartie, ces remises en cause produisent une évolution du rapport entre les coûts de transaction liés aux marchés et ceux liés aux entreprises. On peut donc penser que les nouvelles procédures de contrôle interne vont constituer un enjeu primordial dans la répartition des transactions entre le marché et les entreprises.

\subsection{Les caractéristiques de la nouvelle économie et les coûts de transaction}

Deux caractéristiques de la nouvelle économie sont susceptibles d'avoir des conséquences non négligeables sur les procédures de contrôle interne en vigueur au sein des entreprises.

\subsubsection{Une évolution de la compétence}

Historiquement, deux modèles principaux de qualification des individus ont prédominé. Il s'agit du modèle du métier où la qualification vient sanctionner un long processus d'apprentissage; et du modèle du poste de travail où la qualification des individus dépend du poste de travail qu'ils occupent (Zarifian 2001). Dans le cas du modèle de métier, le contrôle interne est en partie défini par les acteurs eux-mêmes en fonction des exigences de leur métier: "ces métiers (...) reproduisent des apprentissages en réseaux, entre pairs, des appartenances et communications transversales, et autodéfinissent leurs propres critères de jugement sur la qualification et son niveau." (Zarifian 2001, p.15). A l'inverse, dans le cas du modèle du poste de travail, le contrôle interne est défini par une instance extérieure qui utilise l'organisation de ces postes de travail (par exemple à travers la séparation des tâches) pour garantir son contrôle de l'entreprise.

Selon Zarifian, le modèle de la compétence aurait commencé à apparaître dès les années 70. Ce modèle se caractérise par son attention portée aux "compétences" des individus pour faire face à diverses situations que l'on ne pouvait pas nécessairement prévoir ou planifier. Le modèle de la compétence repose donc sur une autonomie accrue accordée aux individus pour prendre des décisions ou des actions en fonction de la situation concrète qu'ils rencontrent: "il 
se produit une problématisation du travail: travailler, c'est s'affronter à un problème et chercher à améliorer les solutions" (Zarifian 2001, P.27).

Les enjeux sont très forts en matière de contrôle interne puisque ce modèle suppose une remise à plat des mécanismes de contrôle interne. En effet, les contrôles de type bureaucratique où les tâches sont nettement définies deviennent totalement inadéquats et se retrouvent en contradiction avec l'objectif affiché d'autonomie et de responsabilisation des salariés.

\subsubsection{L'accroissement des connaissances et la spécialisation des savoirs}

L'accroissement exponentiel des connaissances a entraîné l'apparition et le développement des experts, c'est à dire des individus spécialisés dans un domaine précis mais de disposant plus de connaissances suffisamment générales pour couvrir toute l'étendue du savoir. Dans les entreprises, cette situation a conduit à remplacer des individus supposés disposer de telles compétences générales par des équipes constituées de spécialistes (ou d'experts) des différents domaines requis (Cusumano et Nobeoka 1999). Le domaine automobile a sans doute été l'un des domaines précurseurs, puisque si un véhicule pouvait se concevoir au milieu du XXe siècle au sein d'un bureau d'études regroupant des ingénieurs, il n'en est plus de même à la fin du XXe siècle, et l'équipe chargée de la conception et du développement d'un véhicule doit non seulement intégrer des ingénieurs (eux-mêmes spécialisés dans différents domaines), mais également des techniciens de production, des commerciaux et des financiers. 
Schéma 3 : Nouvelle économie et procédures de contrôle interne

Caractéristiques

Incidences

Développement du travail par projet et non plus par fonction

\begin{tabular}{l} 
Incidences \\
\hline $\begin{array}{l}\text { Rattachement d'un même salarié à } \\
\text { plusieurs responsables hiérarchiques } \\
\text { Regroupement sous une même autorité } \\
\text { de salariés ayant des compétences } \\
\text { diverses et appartenant initialement à } \\
\text { différentes fonctions }\end{array}$ \\
\hline
\end{tabular}
Automatisation des procédures de transfert de données: développement des nouvelles technologies de l'information et de la communication

Suppression de certains postes de travail administratifs

Remise en cause du principe de séparation des tâches

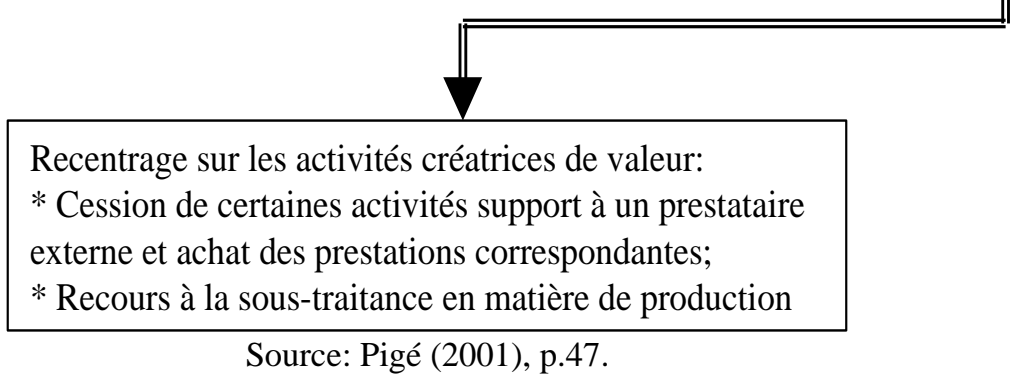

En matière de procédures de contrôle interne, cela a eu pour effet de remettre en cause la règle, édictée par Fayol (1916) au début du siècle, de rattachement d'un salarié à un seul supérieur hiérarchique. En effet, si un salarié continue d'appartenir à une fonction précise, et est rattaché à ce titre à son directeur fonctionnel, il peut être mis à disposition d'une équipe de développement et ainsi répondre aux demandes du directeur de l'équipe. Les problèmes de conduite opportuniste qui peuvent en découler sont bien connus, le salarié étant, soit pris en tenaille entre des ordres contradictoires, soit au contraire libre de jouer de l'un de ses supérieurs par rapport à l'autre. Cela a pour effet mécanique d'accroître les coûts correspondants, et donc de rendre l'entreprise potentiellement moins compétitive que le marché en termes de coûts de transaction.

Selon Midler (1996, pp.134-135): "Alors que l'organisation traditionnelle voit le flux d'activités distribué du haut du métier vers le bas, tous les processus actuels visent à inverser les flux, la charge étant définie à la base. (...) Pour dépasser ce problème, les Directions de projets ont été amenées à instituer d'autres dispositifs de régulation, au niveau de l'encadrement intermédiaire, pour s'assurer de la cohérence des politiques métiers et des engagements à la base. Ainsi se sont structurés progressivement trois niveaux de transversalité projet: d'abord au sommet, puis à la base et enfin avec la hiérarchie 
intermédiaire". Il devient ainsi nécessaire de concilier une logique de métier avec une logique de compétence. Le système de contrôle interne ne peut plus s'appuyer uniquement sur la maîtrise du métier pour s'assurer que l'organisation est bien maîtrisée et contrôlée.

\subsubsection{Le développement des nouvelles technologies de l'information et de la communication}

La communication devient désormais une composante essentielle du travail: la qualité des interactions est centrale pour améliorer la performance des organisations (Zarifian 1999). Si le développement des nouvelles technologies de l'information et de la communication (NTIC) est qualifié de nouvelle révolution, ses effets sont néanmoins encore difficilement appréhendés. On peut cependant essayer d'en mettre en évidence un certain nombre ayant des conséquences non négligeables sur les procédures de contrôle interne.

Les NTIC reposent sur une transmission beaucoup plus rapide de l'information, liée entre autres à la suppression de certaines tâches d'enregistrement, et sur la création de gigantesques bases de données permettant, par une saisie unique des données, d'alimenter de façon personnalisée de multiples utilisateurs. L'exemple le plus marquant en est sans doute le développement des services de courtage en ligne sur Internet. L'utilisateur peut intervenir directement sur le marché, en ayant connaissance du carnet des ordres en attente pour chaque valeur qui l'intéresse. Par rapport à la situation antérieure, de nombreux opérateurs administratifs ont été supprimés. Lors du passage d'un marché à la criée à un marché informatisé, les courtiers ont été progressivement éliminés et les opérateurs bancaires pouvaient intervenir directement en Bourse à partir de leurs écrans informatiques. Avec le développement de l'Internet, ce sont ces personnels bancaires qui sont eux-mêmes supprimés puisque l'utilisateur a accès aux mêmes informations que ces derniers.

En matière de contrôle interne, ces disparitions d'intermédiaires peuvent avoir pour effet de remettre en cause le principe de séparation des tâches qui permet de minimiser les risques de fraude, ou même d'erreurs. La suppression des points de passage obligés, si elle permet d'accélérer le traitement de l'information, a pour contrepartie de rendre cette information plus dépendante de la qualité des canaux de transmission, mais aussi de la qualité du signal initialement transmis. Par exemple, si un utilisateur saisit un ordre de vente en bourse de 1000 titres, au lieu des 100 qu'il détient en portefeuille, une fois la transaction validée, il n'a plus aucun recours ${ }^{\text {iv }}$. Dans le système antérieur, son banquier pouvait lui faire remarquer l'anomalie, ou il pouvait s'en apercevoir en rédigeant la confirmation écrite. Les NTIC, en accélérant l'impact de la prise de décision, rendent celle-ci beaucoup plus brutale. 
On peut donc estimer que, du point de vue du contrôle interne, les contrôles préalables à faire porter sur la chaîne de traitement de l'information seront beaucoup plus élevés qu'auparavant, ce qui devrait renchérir d'autant les coûts initiaux d'exécution. En raisonnant en termes comptables, on pourrait dire qu'au sein de l'entreprise, la part des coûts fixes dans le contrôle interne va en s'accroissant par rapport à la part variable (qui dépend du nombre de transactions effectuées).

En contrepartie, sur le marché, les NTIC réduisent très fortement non seulement les coûts d'information, la recherche sur Internet permet d'économiser la consultation d'annuaires et d'encyclopédies, mais également les coûts de rédaction des contrats, car cette rédaction des contrats peut se faire en supprimant de nombreux intermédiaires ${ }^{\mathrm{v}}$. Dans le domaine du football, qui constitue une sphère d'expérimentation intéressante, certains joueurs ont ainsi décidé de supprimer leurs attachés commerciaux (ceux qui négocient leurs contrats) en les remplaçant par un outil de négociation direct sur Internet (par le biais de l'ouverture d'un site personnel).

\subsection{Le recentrage sur les activités créatrices de valeur et les conséquences en matière de contrôle interne}

Les entreprises sont conduites à repenser toute leur logique organisationnelle et à déterminer les activités qu'il convient d'assurer en propre, par rapport à celles qui peuvent efficacement être sous-traitées auprès du marché. Dans la définition des fonctions à conserver, l'existence de procédures de contrôle interne susceptibles d'offrir un avantage compétitif par rapport au marché sera un élément déterminant. Il est ainsi nécessaire d'identifier les zones de transaction à risque pour y apporter la solution la plus adéquate, et déterminer si le risque ne serait pas mieux géré par le recours au marché.

Le recentrage sur les activités créatrices de valeur peut dans certains cas aboutir à l'externalisation d'une partie des processus de contrôle interne. La question centrale est alors celle des limites de cette externalisation. Jusqu'où l'entreprise peut-elle aller en matière d'externalisation sans perdre sa nature même ? De la même manière, la nouvelle définition des compétences clés des entreprises conduit celles-ci à redéfinir leurs activités et à se séparer de pans entiers de production considérés naguère comme étant parties indispensables du groupe. Quelles en sont les conséquences sur le fonctionnement du contrôle interne et en particulier sur les mécanismes reposant sur une certaine participation et adhésion des membres du personnel? 


\subsubsection{L'identification des activités clés de l'entreprise}

L'incidence de l'évolution des coûts de transaction est très forte puisque les entreprises se trouvent confrontées à une concurrence venant non plus d'entreprises similaires, mais d'entreprises fonctionnant sur une organisation totalement différente qui fait beaucoup plus référence aux marchés. Amazon.com n'est pas une entreprise d'édition mais, en même temps, du point de vue de sa valeur de marché, elle est devenue, ou elle est en train de devenir, le premier acteur de la distribution mondiale d'ouvrages. Bertelsmann qui est le premier éditeur allemand et même européen et qui dispose d'une véritable maison d'édition se trouve confronté à une nouvelle concurrence qui ne porte que sur un fragment de son métier ; c'est-àdire sur la distribution aux clients qui court-circuite le réseau traditionnel de libraires.

Le recentrage de l'entreprise sur ses fonctions de base ne touche pas seulement ces fonctions annexes, mais également des fonctions qui, jusqu'à une date récente, étaient considérées comme indissociablement liées à l'exercice du pouvoir de direction. Il en est ainsi des services comptables ou informatiques qui peuvent faire l'objet d'externalisation, c'est à dire de processus de cession à des entreprises spécialisées du secteur qui, moyennant une redevance annuelle, acceptent de fournir en contrepartie les services auparavant fournis en interne.

\subsubsection{L'externalisation du contrôle interne}

Selon Bouquin (1998, p.36-37): "Le contrôle organisationnel (interne et externe) recouvre largement ce que la profession comptable nomme le contrôle interne" et "le contrôle organisationnel est au service des dirigeants (...) Il est conçu pour orienter leurs propres actions, celles de leurs subordonnés et plus spécifiquement pour maîtriser les transactions internes et externes. S'agissant des relations avec les subordonnés, le contrôle organisationnel (que l'on pourrait qualifier d'interne) peut être renforcé par des mécanismes informels comme la culture d'entreprise."

Il n'est donc pas possible, par définition, d'externaliser en totalité la fonction de contrôle interne sinon cela signifierait que l'organisation elle-même n'a plus d'unité et de direction. Par contre, certaines composantes du contrôle interne peuvent être externalisées. Aux Etats-Unis, les cabinets d'audit proposent la fonction supplémentaire d'audit interne en sus de l'audit externe. Alors que l'audit externe est à destination des tiers et en particulier des actionnaires, l'audit interne est pour sa part à destination des dirigeants et dans une moindre mesure du conseil d'administration. Les missions d'audit interne sont donc définies par la direction de l'entreprise qui, au lieu de les faire réaliser par des salariés de l'entreprise, les confient à un 
cabinet extérieur. Cette mission d'audit interne peut même comprendre la fonction de rédaction et de mise en place de procédures de contrôle interne.

Si nous considérons qu'une entreprise se caractérise en particulier par l'existence d'un système de contrôle interne, à partir de quel moment, ou de quelle dose d'externalisation, l'entreprise cesse-t-elle d'exister ? La faillite d'Enron est à cet égard révélatrice puisque cette entreprise avait décidé de confier son audit interne à son cabinet d'audit externe, en l'occurrence Arthur Andersen. Enron est une entreprise de production et de distribution d'énergie qui s'est fortement développé au cours des dernières années en tant qu'acteur majeur des marchés de dérivés sur les contrats d'énergie. L'analyse du mode de fonctionnement d'Enron semble indiquer qu'un des facteurs principaux de sa désintégration est justement la disparition progressive d'un contrôle organisationnel susceptible d'assurer la cohésion entre des intérêts parfois contradictoires. L'objectif majeur des dirigeants (en fait le président du conseil d'administration, le directeur général et le directeur financier) apparait comme ayant été de coller au maximum à l'évaluation du marché en supprimant tous les freins organisationnels susceptibles de ralentir le développement de ses activités.

De par l'existence de ses procédures de contrôle interne, une entreprise ne peut pas connaître un développement exponentiel durable (puisqu'elle doit alors sans cesse réadapter son architecture organisationnelle à des besoins grandissants de gestion des transactions), alors qu'un marché le peut puisqu'il repose sur une multitude d'acteurs réels ou potentiels. Nous estimons ainsi qu'une entreprise peut externaliser certaines fonctions liées à son contrôle interne mais qu'elle doit néanmoins impérativement en concerner la maîtrise pour tout simplement continuer à exister en tant qu'organisation.

\subsubsection{La rupture du lien d'appartenance et la remise en cause du contrôle organisationnel}

Une seconde caractéristique du contrôle interne est que, dans les entreprises, il repose sur une certaine adhésion du personnel. Alors qu'un système bureaucratique est censé ne reposer que sur des procédures impersonnelles préalablement définies (et qui tôt ou tard mènent au blocage du système), les entreprises sont conduites à s'appuyer sur leur personnel afin de disposer d'un minimum de flexibilité susceptible de favoriser une réponse rapide et adaptée à des événements non nécessairement prévus. Ouchi $(1979,1980)$ estime ainsi que le contrôle organisationnel peut reposer sur les contrôles assurés par les salariés grâce à une socialisation de ces derniers. Cependant Ouchi souligne que pour qu'il y ait une adhésion du personnel, il est nécessaire non seulement que les objectifs de l'entreprise soient cohérents 
avec le système de valeur des salariés mais également que ces derniers puissent se situer dans une perspective à long terme, c'est-à-dire qu'ils soient certains de continuer à faire partie de l'entreprise.

La réflexion sur les limites de l'entreprise et sur l'externalisation d'un certain nombre d'activités pose ainsi le problème de la pérennité de ce lien d'appartenance. Jusqu'à présent, les restructurations des grandes entreprises portaient sur la réduction du personnel pour gagner en productivité. Etait donc privilégié le départ en pré-retraite des salariés les plus âgés. Aujourd'hui, l'exemple des grands groupes spécialisés dans les infrastructures et dans les appareils de télécommunication (Alcatel, Nortel, Lucent pour les principaux) montre que la réflexion sur les limites de l'entreprise conduit à externaliser des secteurs entiers liés par exemple à la production pour ne conserver que les activités de conception (bureau d'études) et de commercialisation. Apparaissent ainsi des sous-traitants (Flextronics par exemple) spécialisés dans la gestion d'usine d'assemblage et fabriquant pour le compte des grands groupes précités.

Il semble que peu de réflexions aient porté sur l'impact de ces décisions en matière de contrôle organisationnel ou de contrôle interne. L'entreprise initiale peut-elle conserver un système de contrôle interne identique alors même qu'elle remet en cause de façon permanente les différents liens d'appartenance ? Dans de nombreux sites les salariés sont ainsi privés de repères à la suite des stratégies de recentrage des dirigeants. Pour ne prendre qu'un exemple, le site de Belfort a longtemps été associé aux turbines à vapeur d'Alstom. Ces dernières ont été cédées à Siemens alors que dans le même temps Alstom a repris les turbines à vapeur d'ABB. Les anciens salariés d'Alstom du site de Belfort ont ainsi intégré une entreprise devenue concurrent direct de leur ancien employeur.

Il devient absolument impératif de repenser l'impact des restructurations sur le fonctionnement même du contrôle interne pour appréhender les risques croissants de déresponsabilisation des employés qui ne se perçoivent plus comme appartenant à un groupe véhiculant une culture d'entreprise et son corpus de valeur, mais simplement comme des moyens de production soumis à une loi du marché qui leur échappe. On pourrait ainsi estimer que la faillite d'Enron est d'une certaine manière le résultat de cette absence de notion d'appartenance des employés. En effet, la plupart des transactions incriminées ayant donné lieu à l'annonce de corrections comptables significatives, étaient connues des employés des services chargés de contrôler ces transactions. Pourtant, peu d'entre eux se sont émus des conséquences potentielles pour le groupe, et moins encore ont eu le courage d'en discuter 
avec les dirigeants (le trésorier général MacMahon qui faisait exception a vu son poste transféré à la suite de son entretien avec le directeur général).

\section{Conclusion}

La révolution économique, à laquelle nous sommes en train d'assister, va remettre en cause un certain nombre de principes supposés comme acquis. Parmi ceux-ci, nous mettons en évidence la place essentielle des mécanismes et des procédures de contrôle interne. Ce sont eux qui assurent la cohérence de toute organisation et le maintien de son intégrité.

L'émergence de nouveaux acteurs économiques dont la structure organisationnelle n'est plus, ou plus nécessairement, calquée sur la structure des concurrents en place depuis de nombreuses années, entraîne pour ces derniers, non seulement l'obligation de repenser leurs stratégies et la pertinence de leurs axes de développement, mais aussi et surtout le besoin de repenser les mécanismes et les procédures conduisant à la fiabilité et à la transmission de l'information, qu'il s'agisse d'une information ascendante (information de la direction par les acteurs sur le terrain) ou descendante (décisions à faire appliquer par transmission hiérarchique). Le contrôle interne est donc destiné à se trouver au cœur de toute réflexion sur l'évolution des organisations actuelles et leur confrontation à un environnement concurrentiel dont les règles sont en train de se modifier.

L'évolution de la Bourse semble rythmer l'histoire de la nouvelle économie. Après l'euphorie des années 1999 et 2000, la descente aux enfers des années 2001 et 2002 semblerait condamner la notion de "nouvelle économie" à une brève apparition historique. Les arguments que nous avons exposé au cours de cet article tendent à infirmer cette vision pessimiste. Il nous semble que la redéfinition des structures organisationnelles reste plus que jamais d'actualité même si les feux de la rampe s'en sont un peu éloignés. A contrario, les difficultés que connaissent la plupart des groupes impliqués dans les nouvelles technologies de l'information et de la communication soulignent l'importance des mécanismes de contrôle interne comme garants de la structure organisationnelle par rapport à un marché qui tend à devenir de plus en plus présent.

\section{Bibliographie}

Alchian A.A. (1984): "Specificity, specialization and coalitions", Journal of Economic Theory and Institutions, $\mathrm{N}^{\circ} 140$, March. 
Arrow K.J. (1969): "The Organization of Economic Activity", The analysis and evaluation of public expenditure: the PPBS system. Joint Economic Committee, $91^{\text {st }}$ Congress, $1^{\text {st }}$ session, p.48.

Becour J.C. et Bouquin H. (1996): Audit opérationnel, Economica.

Bouquin H. (1998): Le contrôle de gestion, $4^{\mathrm{e}}$ édition, Gestion PUF.

Coase R.H. (1937): “The nature of the Firm”, Economica, Vol.4, November, pp.331-351.

Collins L. et Valin G. (1992): Audit et contrôle interne, Dalloz.

Coopers \& Lybrand et IFACI (1994): La nouvelle pratique du contrôle interne, Editions d'Organisation.

Cusumano M.A. et Nobeoka K. (1999): Le management multi-projets, Dunod, traduit de l'américain: "Thinking Beyond Lean: How Multi-Project Management is Transforming Product Development at Toyota and Other Companies".

Desreumaux A. (1997): "Structures de l'entreprise", in Encyclopédie de gestion, Economica, 2e édition, pp.31473173.

Fayol H. (1916) : "Administration industrielle et générale", Bulletin de la Société de l'industrie minérale, Dunod, réédité en 1979.

Gabrié H. et Jacquier J.L. (1994): La théorie moderne de l'entreprise, Economica.

Midler C. (1996): L'auto qui n'existait pas, Dunod.

Mikol A. (1991) : "Principes généraux de contrôle interne", Revue Française de Comptabilité, N²19, janvier.

Ouchi W. (1979): "A Conceptual Framework for the Design of Organizational Control", Administrative Science Quarterly, Vol.24, September, pp.833-848.

Ouchi W. (1980): "Markets, Bureaucracies and Clans", Administrative Science Quarterly, Vol.25, March, pp.129-141.

Pigé B. (2001): Audit et contrôle interne, $2^{\mathrm{e}}$ édition, Les Essentiels de la Gestion, EMS.

Renard J. (1997): Théorie et pratique de l'audit interne, éditions d'Organisation.

Williamson O.E. (1985): The Economic Institutions of Capitalism, The Free Press.

Zarifian P. (1999): "Productivité, logique de service et mutations du travail", Revue Française de Gestion, novembre-décembre, pp.106-116.

Zarifian P. (2001): Le modèle de la compétence, Editions Liaisons.

i Extrait d'un tarif de 1990. Le tarif 2000 ne fait plus apparaître qu'un seul prix à la journée. Cependant au-delà de cinq jours, sur demande d'un devis l'entreprise de location consent des tarifs dégressifs. Par exemple, pour un matériel loué $772 \mathrm{~F}$ la journée, le prix proposé descend à $500 \mathrm{~F} /$ jour pour une semaine et même à $370 \mathrm{~F} / \mathrm{jour}$ au-delà. Le principe exposé reste donc valable mais n'est plus affiché de façon transparente pour le client.

ii Les procédures assurant la conservation des actifs sont renforcées par l'existence de procédures de supervision ou de formalisation du déroulement des tâches.

iii On suppose que le prix de base facturé au-delà d'une semaine correspond au coût de financement de l'acquisition et au coût d'utilisation (hors contrôle interne) du matériel que ce soit pour l'entreprise locatrice ou pour l'entreprise utilisatrice.

iv Il y a quelques mois, le marché japonais a d'ailleurs connu une évolution très forte sur l'un de ses compartiments en raison d'une erreur de saisie de l'un des opérateurs (les montants échangés s'exprimant en milliards de yen, une erreur sur le nombre de 0 est moins lisible que pour d'autres monnaies).

${ }^{v}$ Depuis 1997, le fournisseur de routeurs internet Cisco a transféré en ligne $70 \%$ de son soutien à la clientèle, que ce soit dans les manuels de produits, le recrutement des employés, la distribution des logiciels ou l'aide technique. Cette suppression des intermédiaires a permis 
d'économiser un montant annuel estimé à 500 millions de dollars soit $9 \%$ des recettes totales (Meeker Mary, 1998: "Internet retailing report", Morgan Stanley). 